\title{
Systematic investigation of parameters of an electrospinning process of poly(acrylic acid) nanofibres using response surface methodology
}

\author{
SEYED VAHID EBADI, AREF FAKHRALI and DARIUSH SEMNANI* \\ Department of Textile Engineering, Isfahan University of Technology, Isfahan 845156-83111, Iran \\ *Author for correspondence (d_semnani@cc.iut.ac.ir)
}

MS received 9 November 2017; accepted 12 July 2018; published online 23 April 2019

\begin{abstract}
In this study, response surface methodology (RSM) based on the central composite design (CCD) was used for modelling the electrospinning process of polyacrylic acid (PAA) nanofibres, so as to assess simultaneously the effect of the most important electrospinning parameters (concentration of polymer solution, applied voltage, distance between the nozzle and collector and flow rate of solution) on the diameter of electrospun PAA nanofibres. The surface morphology was studied by scanning electron microscopy (SEM). The average diameter of PAA nanofibres obtained was from 233 to $1210 \mathrm{~nm}$ from SEM images with different process parameters. The results showed that the solution concentration, the applied voltage and the distance between the nozzle and collector are, in that order, the most important parameters affecting the diameter of nanofibres. The flow rate, however, showed no significant effect on the nanofiber diameter. The RSM model predicted that under optimal electrospinning conditions (solution concentration of $3 \mathrm{w} / \mathrm{v} \%$, voltage of $16 \mathrm{kV}$, electrospinning distance of $15 \mathrm{~cm}$ and flow rate of $1.75 \mathrm{ml} \mathrm{h}^{-1}$ ), the nanofibres would be $262 \mathrm{~nm}$ in diameter, which was proved to be very close to the actual measured value. Therefore, the obtained results demonstrated the good performance of the RSM model in investigating the effect of electrospinning variables and predicting the diameter of PAA nanofibres. PAA nanofibres have great potential in applications such as sensors and biosensors, removal of heavy metals and contaminants, muscle tissue engineering, etc. and the use of thinner nanofibres leads to their improved performance in these applications.
\end{abstract}

Keywords. Electrospinning; response surface methodology; nanofibres; polyacrylic acid; optimization.

\section{Introduction}

In the past decade, polymeric nanofibres have been used in a wide range of industrial and scientific applications [1,2]. The important properties of these nanostructures, including a very high specific surface area (about 1000 times higher than microfibres), flexibility in surface functionalities and desirable porosity and mechanical properties, have made them a suitable basic structure for many applications, such as tissue engineering [3], drug delivery [4], wound dressing [5,6], nanofibrous yarns for sutures [7-9], filtration [10,11], batteries [12], fuel cells [13], enzyme immobilization [14], sensors [15] and actuators [16]. Nanofibres can be produced through methods such as electrospinning, drawing, template synthesis, phase separation and self-assembly; but the simplicity of the electrospinning process and its ability to produce continuous nanofibres have drawn most of the attention to this technique of nanofibre production $[17,18]$.

While numerous parameters can affect the fibre formation in the electrospinning process, the concentration of polymer solution, applied voltage, distance between nozzle and collector and flow rate of polymer solution are the most important factors affecting the morphology and diameter of the produced nanofibres $[19,20]$. Many applications require nanofibres with a set of specific properties. Hence, precise control of the diameter of electrospun nanofibres is of significant importance, since many of the physical, mechanical and electrical properties of electrospun nanofibres are affected by the nanofibre diameter. For example, in some filtration applications of nanofibres, it has been proven that the filter made with thinner nanofibres is more efficient. Furthermore, the results of several studies on other applications, such as tissue engineering, sensors, batteries and actuators suggest that thinner nanofibres exhibit a better performance [21-25]. Therefore, optimization of nanofibers diameter is essential as one of the most important factor in the final applications.

The use of empirical statistical models is a simple and proper method to evaluate the effect of various electrospinning parameters on the diameter of produced nanofibres. This method allows us to precisely control the nanofibre diameter by using the electrospinning parameters and to predict the outcome for untried conditions. Hence, this method is very helpful for a complete understanding of the electrospinning process and the effect of its different factors [20].

Response surface methodology (RSM) is a set of mathematical and statistical methods for modelling and analysis of problems where a response is affected by several parameters 
and the goal is to optimize this response. RSM has many advantages over conventional methods, the most important of which include the reduced number of required experiments and the ability to study the interaction effects between variables [26]. Hence, recent years have witnessed the growing use of this method to optimize the diameter and morphology of electrospun nanofibres. When selecting the response surface design, one should always consider the rotatability properties. This is because in RSM optimization, the location of the optimal point is unknown at the onset of the process, so it is reasonable to use a design with equal precision of estimation along all directions. The most famous rotatable RSM design is the central composite design (CCD). A CCD consists of a $2^{k}$ factorial (or fractional factorial) design with $n_{f}$ factorial points, $2 k$ axial points $( \pm \alpha, 0,0, \ldots, 0)$, $(0, \pm \alpha, 0, \ldots, 0),(0,0, \pm \alpha, \ldots, 0), \ldots,(0,0,0, \ldots, \pm \alpha)$, and $n_{\mathrm{c}}$ centre points $(0,0,0, \ldots, 0)$. The CCD can become rotatable by selecting a suitable $\alpha\left(\alpha=\left(n_{f}\right)^{1 / 4}\right)$. These designs are used to estimate the coefficients of the fitted quadratic model [26].

Recently, RSM has been used to optimize the diameter of electrospun nanofibres made of various polymers, such as polyvinyl alcohol (PVA) [27], polyacrylonitrile (PAN) [28], polymethylmethacrylate (PMMA) [29], polyvinylpyrrolidone (PVP) [30], polyamide 6 (PA 6) [31], etc. [32-34].

Polyacrylic acid (PAA) is a weak anionic polyelectrolyte consisting of carboxylic groups; and the hydrogel network formed from this polymer can absorb water many times more than its weight [35]. This polymer has many applications including filtration, ion exchange resins, membranes for haemodialysis and controlled drug delivery [36]. Recently, the nanofibres made of PAA polymer have attracted much attention in various applications. For example, PAA nanofibres can be used to anchor biomacromolecules, such as enzymes and DNA which is required for bioelectronics and biosensor applications [37]. Also, there have been some research studies on the applications of these nanofibres in sensors [22], removal of heavy metals [38], removal of pollutants [39], nanocomposites [40], actuators [41-43] and muscle tissue engineering [44]. Therefore, the production of very thin and uniform PAA nanofibres with controllable properties can be valuable for many applications.

To the best of our knowledge, there is no report on the use of RSM for evaluating the effects of electrospinning parameters on the diameter of electrospun PAA nanofibres and optimizing the electrospinning conditions to achieve the desired diameter. This study is the first systematic investigation on the simultaneous effect of four important electrospinning parameters (including concentration of polymer solution, applied voltage, distance between nozzle and collector, and flow rate of polymer solution) on the mean diameter of PAA nanofibres. The aim of this study is to fit an empirical model to experimental data and use it to determine the relationship between electrospinning variables and the diameter of PAA nanofibres. The secondary objective of this work is to predict the optimum conditions for minimizing the diameter of the produced nanofibres.

\section{Experimental}

\subsection{Materials}

PAA $\left(M_{\mathrm{v}}=450,000\right)$ was purchased from Sigma Aldrich. Also, ethanol was obtained from Merck and used as the solvent.

\subsection{Solution preparation and electrospinning}

PAA powder was dissolved in ethanol solvent by using a magnetic stirrer for $8 \mathrm{~h}$ at room temperature and a constant relative humidity of $35 \%$, and different concentrations of PAA were prepared $(2,3,4,5$ and $6 \mathrm{w} / \mathrm{v} \%)$. The home-made electrospinning set-up consisted of a high-voltage DC power supply, a syringe pump (top syringe pump Top-5300) with a needle tip (22 gauge, length $=34 \mathrm{~mm}$, outer diameter $=0.7 \mathrm{~mm}$, inner diameter $=0.4 \mathrm{~mm}$ ) and a collector which was ground and covered with aluminum foil. The electrospinning parameters including PAA concentration, applied voltage, distance between nozzle and collector and flow rate of polymer solution were set at different values to evaluate their effects on the diameter of produced nanofibres. The electrospinning for all samples was performed at a temperature of $25^{\circ} \mathrm{C}$ and a constant relative humidity of $35 \%$.

\subsection{Characterization}

Scanning electron microscopy (SEM, XL-30, Philips) was used to investigate the surface morphology of nanofibrous samples after coating the samples with a thin layer of gold (for $2 \mathrm{~min}$ ) using a sputtering apparatus (Bal-Tec SCD 005 sputter coater) at a temperature of $25^{\circ} \mathrm{C}$ and a constant relative humidity of $35 \%$. The average diameters of nanofibres were obtained by measuring the diameters of 100 randomly selected nanofibres from SEM images (by using SEM images of different sections of samples) employing ImageJ software (National Institute of Health, USA).

\subsection{Experimental design}

This part of work consists of three stages which include conducting the designed statistical experiments, finding a mathematical model with good fit to predict the response, and evaluating the performance of a model in prediction of data. In this study, a CCD was used to fit a quadratic model to investigate the effect of four independent electrospinning variables of concentration $(X 1, \mathrm{w} / \mathrm{v} \%)$, applied voltage $(X 2$, $\mathrm{kV})$, distance between nozzle and collector $(X 3, \mathrm{~cm})$ and flow rate of polymer solution $\left(X 4, \mathrm{ml} \mathrm{h}^{-1}\right)$ on the diameter of PAA nanofibers. Table 1 shows the variables used in the experimental design along with their actual and coded levels. Here, the number of $\alpha$ is 2 , and the CCD consists of 30 empirical experiments. The number of experimental trials has been obtained from the equation $N=2^{k}+2 k+n_{\mathrm{c}}$, where $k$ is the number 
Table 1. Variables and their actual and coded levels for CCD.

\begin{tabular}{lcrrrr}
\hline & & \multicolumn{3}{c}{ Coded levels } \\
\cline { 3 - 6 } Independent variable & Symbol & $-\alpha$ & -1 & 0 & +1 \\
\hline PAA concentration (w/v \%) & $\mathrm{A}$ & 2 & 3 & 4 & 5 \\
Applied voltage (kV) & $\mathrm{B}$ & 10 & 12 & 14 & 16 \\
Distance $(\mathrm{cm})$ & $\mathrm{C}$ & 10 & 15 & 20 & 25 \\
Flow rate $\left(\mathrm{ml} \mathrm{h}^{-1}\right)$ & $\mathrm{D}$ & 1 & 1.25 & 1.5 & 1.75 \\
\hline
\end{tabular}

Table 2. Experimental design and results of CCD.

\begin{tabular}{|c|c|c|c|c|c|c|c|c|c|}
\hline \multirow[b]{2}{*}{ Run } & \multicolumn{4}{|c|}{ Coded values } & \multicolumn{4}{|c|}{ Actual values } & \multirow[b]{2}{*}{ Nanofibre diameter $(\mathrm{nm})$} \\
\hline & $x_{1}$ & $x_{2}$ & $x_{3}$ & $x_{4}$ & $\mathrm{~A}(\mathrm{w} / \mathrm{v} \%)$ & $\mathrm{B}(\mathrm{kV})$ & $\mathrm{C}(\mathrm{cm})$ & $\mathrm{D}\left(\mathrm{ml} \mathrm{h}^{-1}\right)$ & \\
\hline 1 & 1 & -1 & 1 & -1 & 5 & 12 & 25 & 1.25 & 966 \\
\hline 2 & 1 & 1 & -1 & 1 & 5 & 16 & 15 & 1.75 & 600 \\
\hline 3 & 0 & 0 & 0 & 2 & 4 & 14 & 20 & 2 & 519 \\
\hline 4 & 2 & 0 & 0 & 0 & 6 & 14 & 20 & 1.5 & 1210 \\
\hline 5 & -1 & 1 & -1 & 1 & 3 & 16 & 15 & 1.75 & 245 \\
\hline 6 & 1 & -1 & 1 & 1 & 5 & 12 & 25 & 1.75 & 1023 \\
\hline 7 & 1 & -1 & -1 & 1 & 5 & 12 & 15 & 1.75 & 824 \\
\hline 8 & 0 & 0 & 0 & 0 & 4 & 14 & 20 & 1.5 & 485 \\
\hline 9 & 0 & -2 & 0 & 0 & 4 & 10 & 20 & 1.5 & 695 \\
\hline 10 & 1 & 1 & 1 & 1 & 5 & 16 & 25 & 1.75 & 703 \\
\hline 11 & 0 & 0 & 0 & 0 & 4 & 14 & 20 & 1.5 & 570 \\
\hline 12 & 0 & 0 & 0 & 0 & 4 & 14 & 20 & 1.5 & 501 \\
\hline 13 & 0 & 0 & 2 & 0 & 4 & 14 & 30 & 1.5 & 550 \\
\hline 14 & 0 & 0 & 0 & -2 & 4 & 14 & 20 & 1 & 498 \\
\hline 15 & -1 & -1 & -1 & -1 & 3 & 12 & 15 & 1.25 & 306 \\
\hline 16 & 1 & 1 & 1 & -1 & 5 & 16 & 25 & 1.25 & 690 \\
\hline 17 & 0 & 0 & 0 & 0 & 4 & 14 & 20 & 1.5 & 518 \\
\hline 18 & -1 & -1 & -1 & 1 & 3 & 12 & 15 & 1.75 & 337 \\
\hline 19 & -1 & -1 & 1 & 1 & 3 & 12 & 25 & 1.75 & 410 \\
\hline 20 & -1 & 1 & 1 & 1 & 3 & 16 & 25 & 1.75 & 297 \\
\hline 21 & -1 & 1 & 1 & -1 & 3 & 16 & 25 & 1.25 & 309 \\
\hline 22 & -1 & 1 & -1 & -1 & 3 & 16 & 15 & 1.25 & 284 \\
\hline 23 & 0 & 0 & -2 & 0 & 4 & 14 & 10 & 1.5 & 413 \\
\hline 24 & 1 & 1 & -1 & -1 & 5 & 16 & 15 & 1.25 & 624 \\
\hline 25 & 0 & 2 & 0 & 0 & 4 & 18 & 20 & 1.5 & 395 \\
\hline 26 & 1 & -1 & -1 & -1 & 5 & 12 & 15 & 1.25 & 748 \\
\hline 27 & -1 & -1 & 1 & -1 & 3 & 12 & 25 & 1.25 & 388 \\
\hline 28 & -2 & 0 & 0 & 0 & 2 & 14 & 20 & 1.5 & 233 \\
\hline 29 & 0 & 0 & 0 & 0 & 4 & 14 & 20 & 1.5 & 524 \\
\hline 30 & 0 & 0 & 0 & 0 & 4 & 14 & 20 & 1.5 & 566 \\
\hline
\end{tabular}

of factors $(=4)$ and $n_{\mathrm{c}}$ is the number of center points $(=6)$ for the estimation of pure error. Table 2 shows the experimental design used in this study.

The first step in RSM is to find an appropriate approximation for the relationship between the response and independent variables. In this step, the common practice is to use a loworder polynomial in some regions of independent variables. The statistical significance of the model is assessed through the use of analysis of variance (ANOVA). It is also imperative to make sure of the validity of the model. In this 


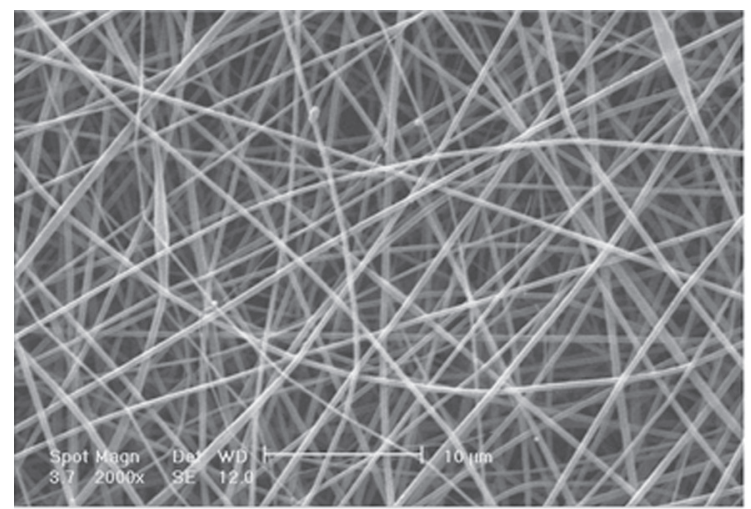

$\mathrm{A}=3 \mathrm{w} / \mathrm{v} \%, \mathrm{~B}=12 \mathrm{kV}, \mathrm{C}=15 \mathrm{~cm}, \mathrm{D}=1.75 \mathrm{ml} / \mathrm{h}$

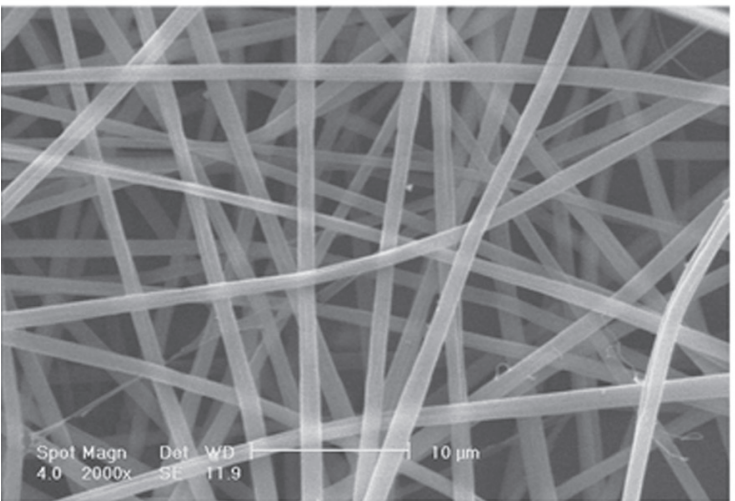

$\mathrm{A}=5 \mathrm{w} / \mathrm{v} \%, \mathrm{~B}=12 \mathrm{kV}, \mathrm{C}=25 \mathrm{~cm}, \mathrm{D}=1.25 \mathrm{ml} / \mathrm{h}$

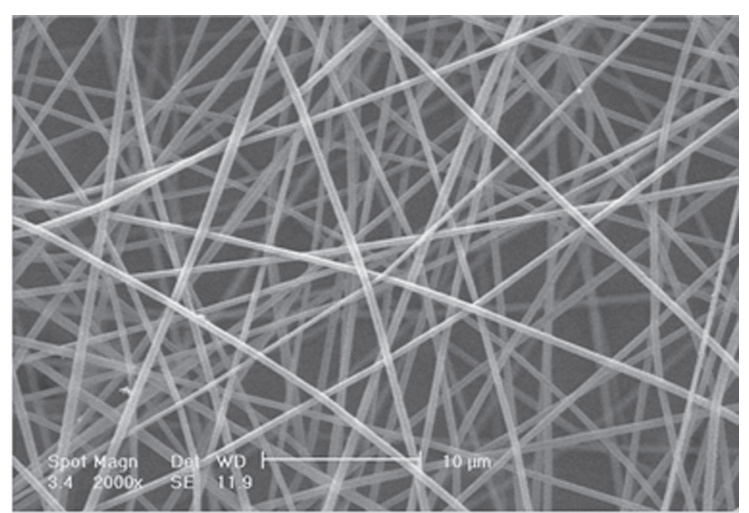

$\mathrm{A}=3 \mathrm{w} / \mathrm{v} \%, \mathrm{~B}=12 \mathrm{kV}, \mathrm{C}=25 \mathrm{~cm}, \mathrm{D}=1.75 \mathrm{ml} / \mathrm{h}$

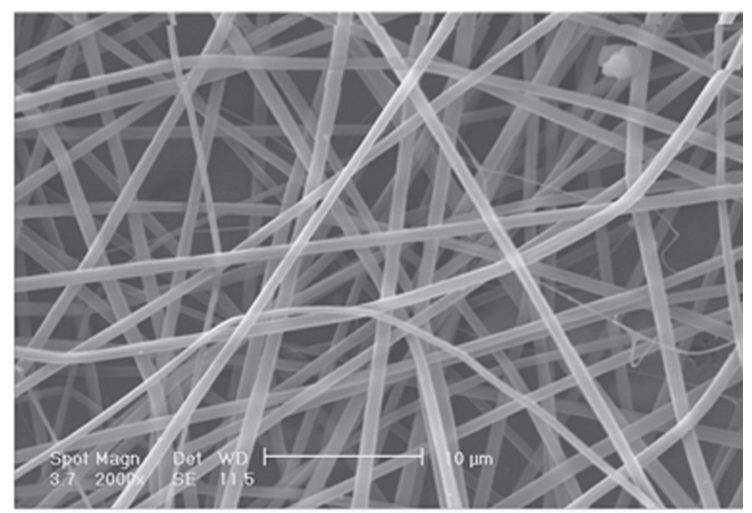

$\mathrm{A}=5 \mathrm{w} / \mathrm{v} \%, \mathrm{~B}=16 \mathrm{kV}, \mathrm{C}=25 \mathrm{~cm}, \mathrm{D}=1.75 \mathrm{ml} / \mathrm{h}$

Figure 1. SEM images of typical PAA electrospun nanofibres.

Table 3. ANOVA table for response surface quadratic model.

\begin{tabular}{lcccccc}
\hline Source & Sum of squares & df & Mean square & $F$ value & $P$ value & Status \\
\hline Model & 1597610 & 14 & 114115 & 127.2107 & $<0.0001$ & Significant \\
A, concentration & 1286214 & 1 & 1286214 & 1433.818 & $<0.0001$ & Significant \\
B, voltage & 142604.2 & 1 & 142604.2 & 158.9692 & $<0.0001$ & Significant \\
C, distance & 49686 & 1 & 49686 & 55.38787 & $<0.0001$ & Significant \\
D, flow rate & 1148.167 & 1 & 1148.167 & 1.279928 & 0.2757 & - \\
AB & 25440.25 & 1 & 25440.25 & 28.35973 & $<0.0001$ & Significant \\
AC & 7832.25 & 1 & 7832.25 & 8.731065 & 0.0098 & Significant \\
AD & 900 & 1 & 900 & 1.003282 & 0.3324 & - \\
BC & 6642.25 & 1 & 6642.25 & 7.404502 & 0.0158 & Significant \\
BD & 3844 & 1 & 3844 & 4.28513 & 0.0561 & - \\
CD & 81 & 1 & 81 & 0.090295 & 0.7679 & - \\
$\mathrm{A}^{2}$ & 57305.19 & 1 & 57305.19 & 63.88143 & $<0.0001$ & Significant \\
$\mathrm{B}^{2}$ & 68.7619 & 1 & 68.7619 & 0.076653 & 0.7857 & - \\
$\mathrm{C}^{2}$ & 5602.333 & 1 & 5602.333 & 6.245247 & 0.0246 & Significant \\
$\mathrm{D}^{2}$ & 1560.048 & 1 & 1560.048 & 1.739076 & 0.2070 & - \\
Residual & 13455.83 & 15 & 897.0556 & - & - & - \\
Lack of fit & 7556.5 & 10 & 755.65 & 0.640454 & 0.7437 & Not significant \\
Pure error & 5899.333 & 5 & 1179.867 & - & - & - \\
Corr. total & 1611066 & 29 & - & - & - & - \\
\hline
\end{tabular}


work, the approximating function is the following quadratic model:

$$
\begin{aligned}
Y= & \beta_{0}+\sum_{i=1}^{k} \beta_{i} x_{i}+\sum_{i=1}^{k} \beta_{i i} x_{i}^{2} \\
& +\sum_{i=1}^{k-1} \sum_{j=i+1}^{k} \beta_{i j} x_{i} x_{j},
\end{aligned}
$$

where $Y$ is the predicted response, $x_{i}$ and $x_{j}$ are the coded values of the independent variables, $\beta_{0}$ is the intercept, $\beta_{i}$ is

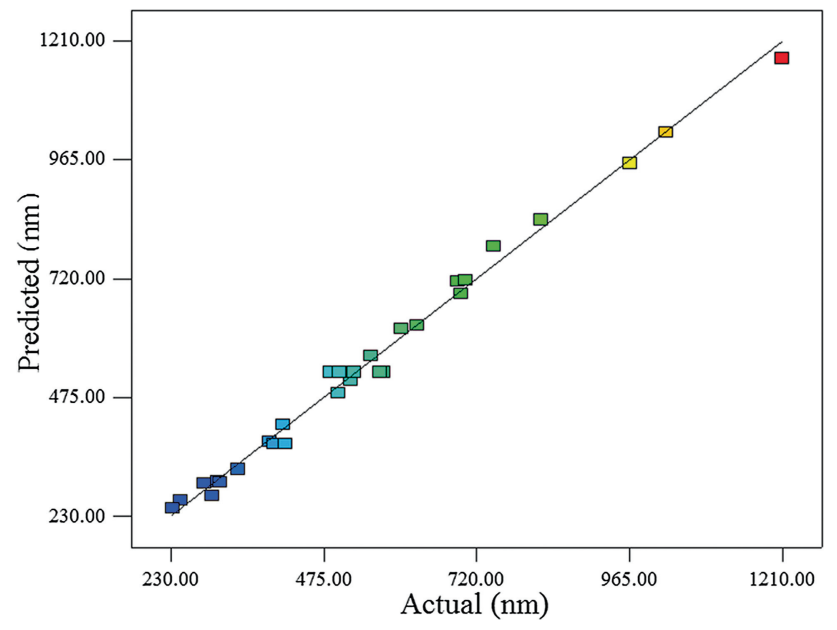

Figure 2. Actual response values (diameter of nanofibres) vs. the predicted response values.

(a)

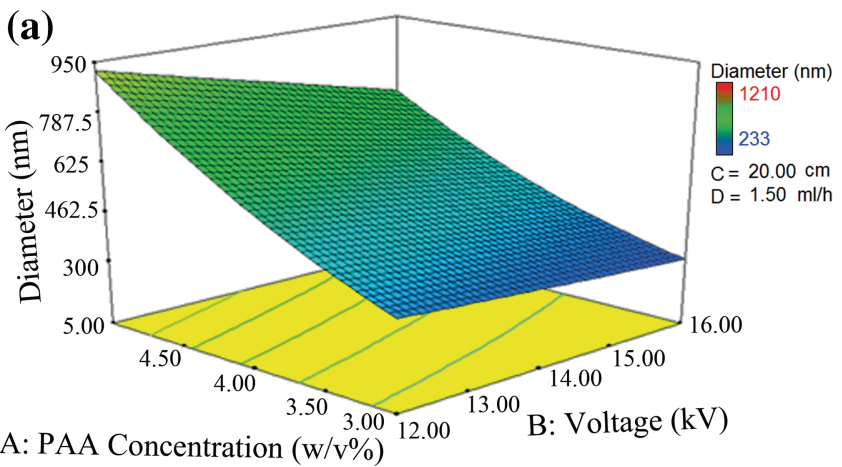

the $i$-th linear coefficient, $\beta_{i j}$ is the $i j$-th interaction coefficient and $\beta_{i i}$ is the $i$-th quadratic coefficient [26].

The Design-Expert software package (version 7.0.0) was used for regression analysis and plotting surface responses. All experiments were performed in a random order.

\section{Results and discussion}

\subsection{Nanofibre morphology}

First, a preliminary study was conducted by the use of SEM images to assess the possibility of producing PAA nanofibres under various conditions. The selected range of electrospinning variables was based on these observations and jet stability conditions. Figure 1 shows the SEM images of typical electrospun PAA nanofibres. As can be seen, the electrospun nanofibres produced under different conditions are beadless and have a smooth and uniform surface morphology. Other samples also had a desirable surface morphology.

\subsection{Estimation of quadratic polynomial model}

Analysis of variance (ANOVA) performed for the quadratic polynomial model is shown in table 3 . The $P$ values $<0.05$ represent the significant of the term, while the $P$ values $>0.05$ are not significant. The model $F$-value of 127.21 with a (b)

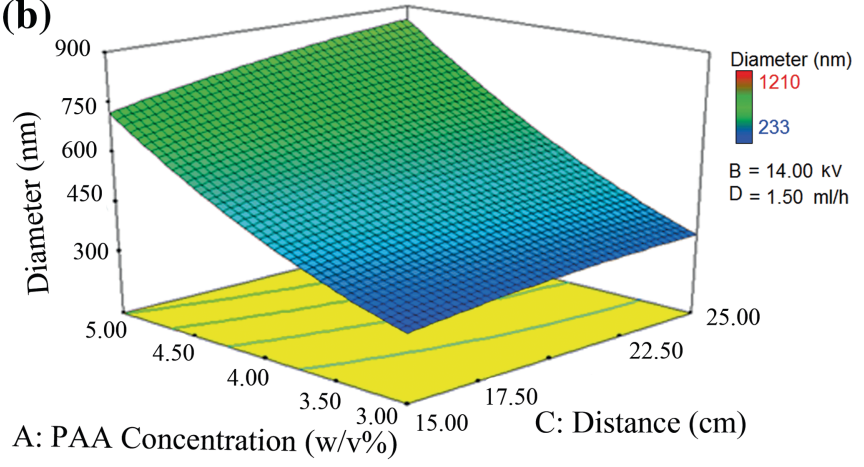

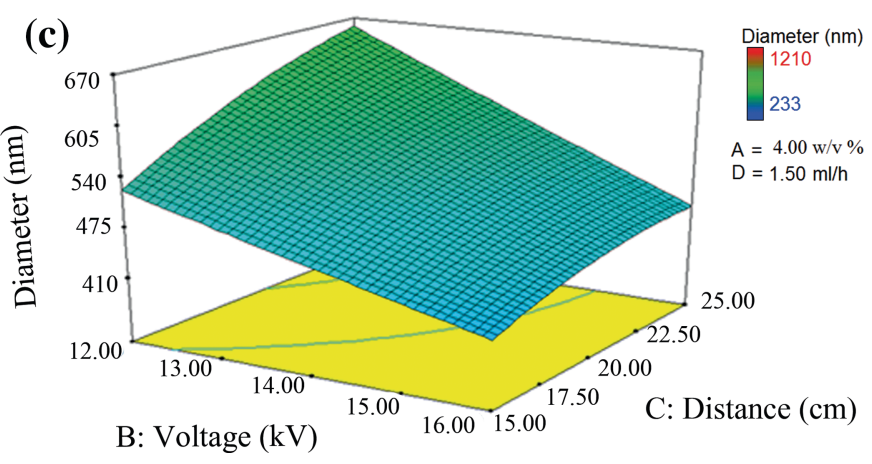

Figure 3. Response surface plots on the nanofibre diameter: effect of (a) PAA concentration and voltage, (b) PAA concentration and distance and (c) voltage and distance (in all the plots other variables are held at centre levels). 

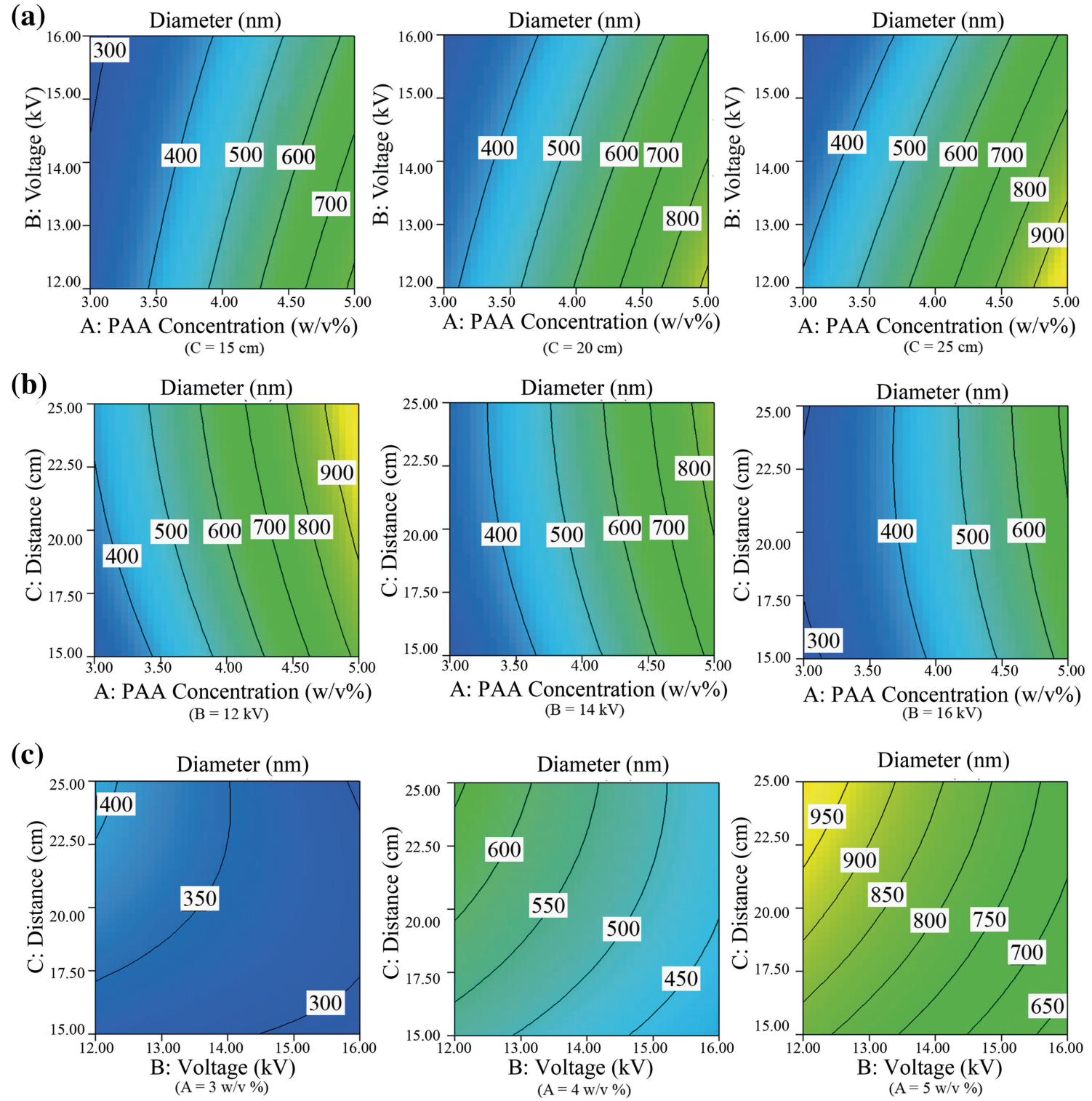

Figure 4. Contour plots of the average diameter of PAA nanofibres as a function of (a) concentration and voltage, (b) concentration and distance and (c) voltage and distance, at three different levels of the third effective variable.

very low model $P$ value $(<0.0001)$ implies that the model is significant. Also the lack of fit $F$-value of 0.64 implies that the lack of fit is not significant relative to the pure error.

Therefore, the second-order model is an adequate approximation to the actual surface. The efficiency of the obtained model was investigated by the coefficient of determination $\left(R^{2}\right)$. In this case, the value of the determination coefficient $\left(R^{2}=0.9677\right)$ indicates that only $3.23 \%$ of the total variations are not explained by the model. Also, the high value of the adjusted determination coefficient (Adj. $R^{2}=0.9839$ ) represents a high significance of the model. After determining the coefficients, the fitted second-order model in terms of coded variables is given by:

$$
\begin{aligned}
Y= & 527.33+231.50 x_{1}-77.08 x_{2}+45.50 x_{3}+6.92 x_{4} \\
& -39.88 x_{1} x_{2}+22.12 x_{1} x_{3}+7.50 x_{1} x_{4}-20.37 x_{2} x_{3} \\
& -15.50 x_{2} x_{4}+2.25 x_{3} x_{4}+45.71 x_{1}^{2}+1.58 x_{2}^{2} \\
& -14.29 x_{3}^{2}-7.54 x_{4}^{2} .
\end{aligned}
$$

The results of analysis of variance show that the terms, $x_{4}$, $x_{1} x_{4}, x_{2} x_{4}, x_{3} x_{4}, x_{2}^{2}$ and $x_{4}^{2}$ are not significant $(P$ value $>0.05)$. Hence, use of RSM leads to the final empirical model shown below which indicates the relationship between the 

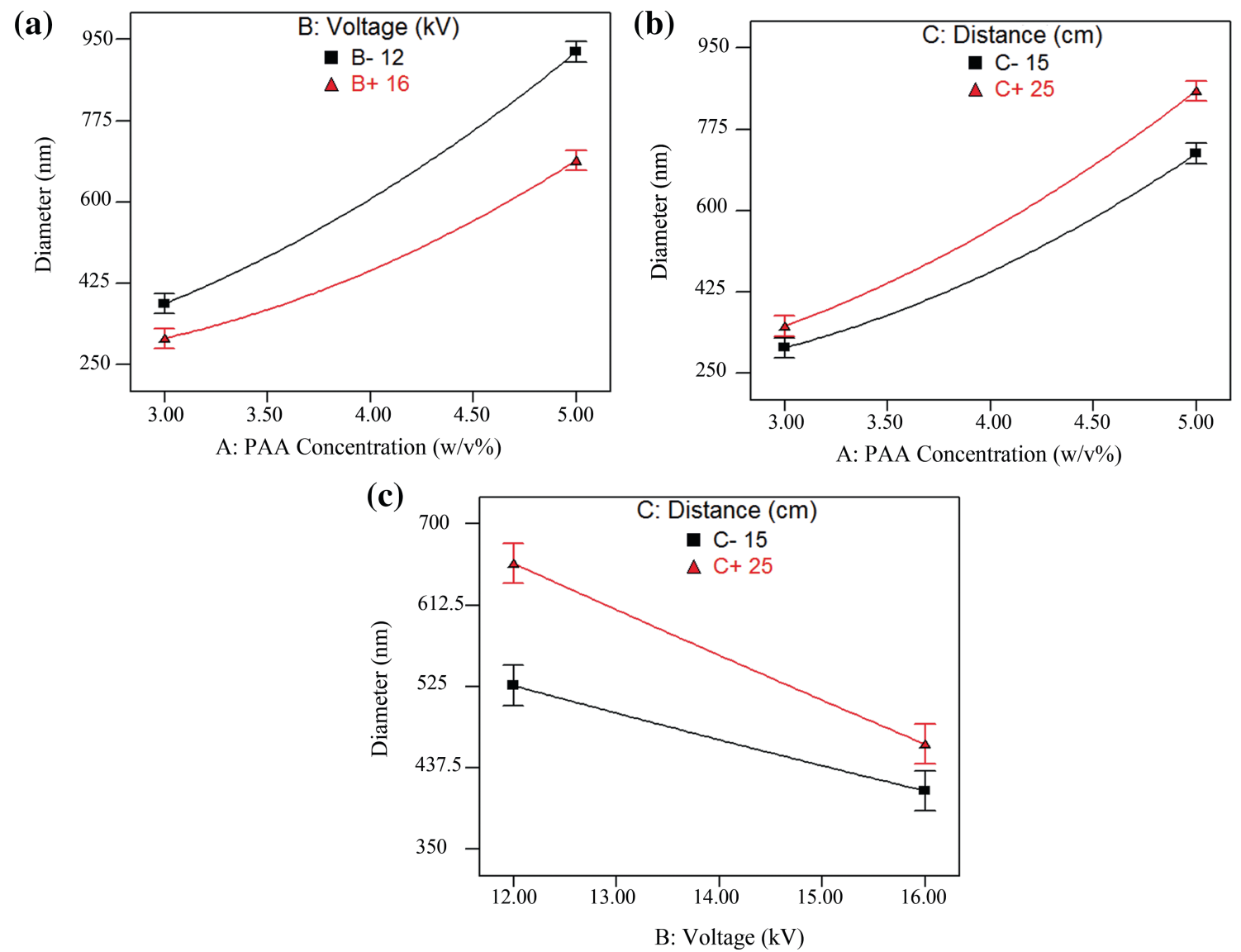

Figure 5. Interaction plots for the effect of different parameters on the diameter of PAA nanofibres: (a) concentration and voltage, (b) concentration and distance and (c) voltage and distance (in all the plots other variables are held at centre levels).

diameter of electrospun PAA nanofibres and coded electrospinning variables:

$$
\begin{aligned}
Y= & 527.33+231.50 x_{1}-77.08 x_{2}+45.50 x_{3}-39.88 x_{1} x_{2} \\
& +22.12 x_{1} x_{3}-20.37 x_{2} x_{3}+45.71 x_{1}^{2}-14.29 x_{3}^{2} .
\end{aligned}
$$

Figure 2 shows the plot of the actual response values (diameter of nanofibres) vs. the predicted response values. This graph is useful to detect a value, or group of values, that are not easily predicted by the model. As can be seen, there is a good agreement between experimental data and predicted values by a model.

\subsection{Effects of variables on average diameter of nanofibres}

The effects of concentration of polymer solution, applied voltage, distance between nozzle and collector, and flow rate on the diameter of PAA nanofibres were examined. Equation (3) shows that both the concentration of polymer solution and the distance between nozzle and collector have a direct relationship with the diameter of electrospun nanofibres. There is also an indirect relationship between the applied voltage and the diameter of nanofibres. Overall, the results show that the concentration of polymer solution has the greatest impact on the diameter of the nanofibres and the flow rate has no significant effect on the diameter.

The predicted model is visualized with the use of response surfaces and contour plots. The response surface presents the response as a surface plotted in three-dimensional space based on two independent variables. The contour plots can be used to simplify the visualization of the response surface. These plots show the lines of the constant response in a two-dimensional plane of two independent variables. The contour plots provide valuable information about the fitted model, but may not represent the complete behaviour of the system. It should be noted that contours (or surfaces) represent contours of the estimated response, and the general nature of the system will be extracted as a result of the fitted model [45].

Figure 3 shows the diameter of nanofibres as the predicted response in the form of three-dimensional response surfaces produced by the fitted model. Also, the two-dimensional contour plots are shown in figure 4. All contour plots show the effects of two independent variables on the diameter of the PAA nanofibres at three different levels of the third effective variable. In all the investigations, the flow rate, which exhibited no significant effect on the response, was fixed on the centre level. 
The results show that one of the most important factors affecting the diameter of the PAA nanofibres is the concentration of polymer solution. By increasing the concentration of polymer solution, a significant increase in the diameter of electrospun nanofibres was observed. An increased concentration of polymer solution leads to increased polymer chain entanglements and increased solution viscosity. Therefore, the viscoelastic force and the jet resistance against the electrostatic stretching force increased, which led to increased diameter of the electrospun PAA nanofibres in the electrospinning process as observed in other studies [30,46]. Another important factor in the process of electrospinning is the level of the applied voltage, so that applying a higher voltage causes a decrease in the diameter of PAA nanofibres. The use of a higher voltage increases the strength of electric field and leads to exertion of a greater stretching force on the electrospinning jet, which in turn decreases the resulting diameter. The third factor affecting the diameter of the PAA nanofibres is the distance between nozzle and collector. The results for smaller distances show that the PAA nanofibres obtained are thinner. By increasing this parameter, the diameter of the produced nanofibres increased. The reason for this observation is that when this distance increases, the strength of the electric field decreases. This phenomenon leads to exertion of a smaller stretching force on the jet and increased diameter of the produced nanofibres. These observations are consistent with other results [20]. In addition, contour plots of figures 4 and 5 show the interaction effects between variables. In all these plots, the presence of nonparallel lines confirms the interaction between the concentration of polymer solution, the applied voltage and the distance between nozzle and collector. The existence and significance of terms, $x_{1} x_{2}, x_{1} x_{3}$ and $x_{2} x_{3}$ in the predicted model for the diameter of PAA nanofibres (equation 3 ) also confirms these observations.

When the applied voltage is low, the concentration of polymer solution has a greater effect on the diameter of nanofibres. As mentioned earlier, the increased concentration of polymer solution leads to increased polymer chain entanglements and thereby increased jet resistance against the electrostatic stretching force. At lower voltages (weaker electric field strength), the weaker electrostatic stretching force can more easily be overcome by the viscoelastic force of the jet, and this causes the effect of concentration on the diameter to become more pronounced $[20,47]$. But at high voltages, the concentration has a reduced effect. The interaction between the polymer solution concentration and the distance between nozzle and collector can be explained with a similar argument: when the nozzle-collector distance is greater, the effect of the electric field is weaker and for the same reasons previously stated for the voltage, the effect of polymer solution concentration is more pronounced.

The importance of the distance between nozzle and collector can be studied from two perspectives: (i) its effect on the strength of the electric field and (ii) its effect on the time of applying the electric field on the jet and the exerted stretching force. In other words, the strength of the electric field is the limiting factor at great distances, and the time of applying the electric field is the limiting factor at small distances. Therefore, at greater nozzle-collector distances, when the electric field strength is weak and it is applied for a longer time, the effect of the applied voltage is more pronounced. Also, at low voltages, when the strength of the electrical field is the limiting factor, the effect of nozzle-collector distance is more pronounced, because a change in this distance alters the strength of electric field. But at higher voltages when the electric field strength is strong, a change in the nozzle-collector distance has a lesser effect on the diameter of nanofibres. These results are in agreement with the literature data [20].

\subsection{Optimization}

The goal of RSM is to optimize the operating conditions of the system or to determine the region where operating conditions are satisfied. Desirability is a mathematical technique for finding the optimums and it is an objective function whose value ranges from 0 to 1 . Therefore, numerical optimization finds a point that maximizes the desirability function. Here, the goal is to find the optimum conditions for the production of PAA nanofibres with a minimum diameter based on electrospinning parameters. Figure 6 shows the optimum conditions for the production of PAA nanofibres with a minimum diameter. As can be seen, under the optimum conditions, polymer solution has a concentration of $3 \mathrm{w} / \mathrm{v} \%$, the applied voltage is $16 \mathrm{kV}$, the distance between nozzle and collector is $15 \mathrm{~cm}$ and the flow rate is $1.75 \mathrm{ml} \mathrm{h}^{-1}$, and the diameter predicted under this condition is $262 \mathrm{~nm}$ (with desirability of 0.969).

To evaluate the performance of RSM in estimation, the diameter predicted by this method was compared with the diameter of produced nanofibres under the above-mentioned

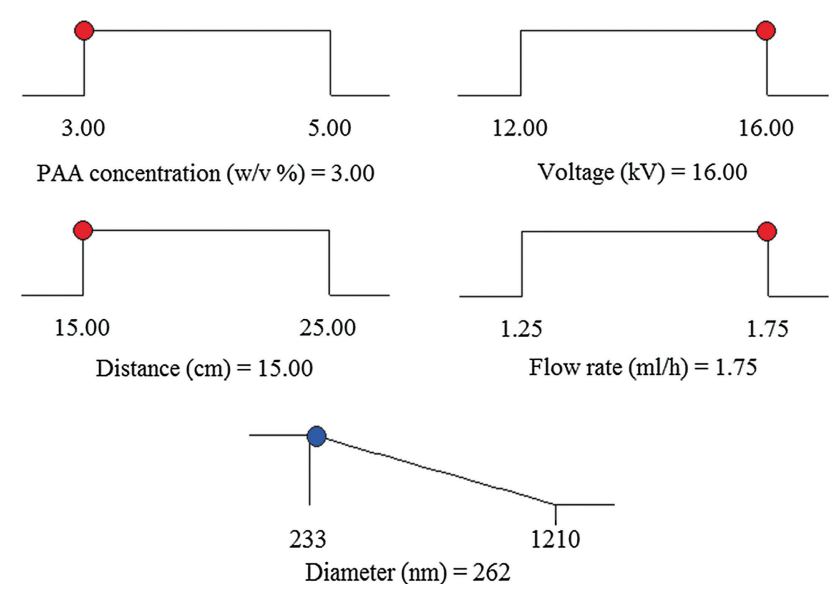

Figure 6. Optimized conditions $(A=3 \mathrm{w} / \mathrm{v} \%, B=16 \mathrm{kV}, C=$ $15 \mathrm{~cm}$ and $D=1.75 \mathrm{ml} \mathrm{h}^{-1}$ ) for producing the PAA nanofibres with a minimum diameter $(262 \mathrm{~nm})$. 


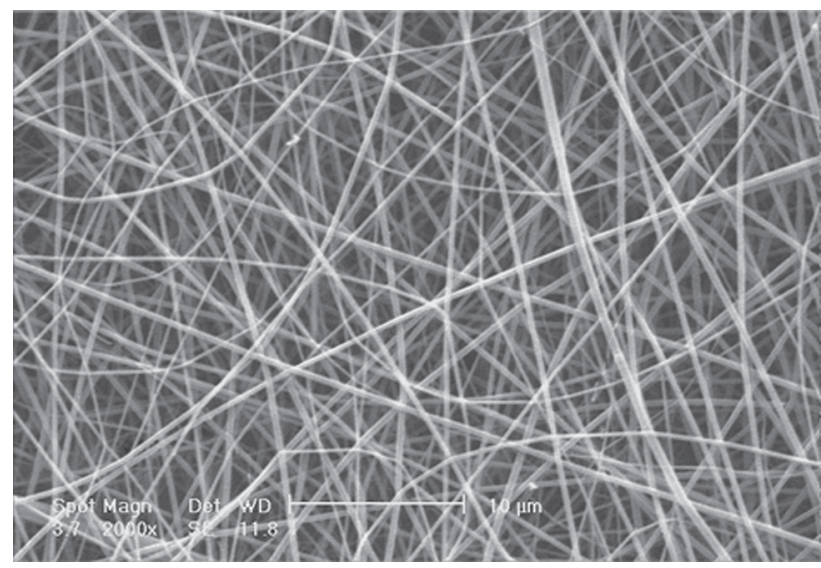

Figure 7. SEM image of the PAA nanofibres produced under optimum conditions: $A=3 \mathrm{w} / \mathrm{v} \%, B=16 \mathrm{kV}, C=15 \mathrm{~cm}$ and $D=1.75 \mathrm{ml} \mathrm{h}^{-1}$.

conditions. According to figure 7 which shows the SEM image of the nanofibres produced under optimum conditions, the average diameter of the nanofibres produced under these conditions is $245 \mathrm{~nm}$. The predicted diameter in the optimization process only has a $6.93 \%$ difference with the value obtained from the SEM image, and this demonstrates the accuracy and precision of the employed method. Compared with previous work, the use of RSM method leads to the production of uniform nanofibres of PAA with a smaller diameter $[22,38,48,49]$. This result is due to the simultaneous systematic investigation of the effect of electrospinning parameters and study of the interaction effects between variables in the RSM [30].

\section{Conclusions}

This study investigated the effect of four main electrospinning parameters on the diameter of PAA nanofibres, and used the CCD technique of RSM for modelling and for optimizing the average diameter of nanofibres. RSM showed that the concentration of polymer solution has the greatest impact on the diameter of the electrospun nanofibres, and increasing the concentration leads to a significant increase in the diameter. The applied voltage and the distance between nozzle and collector were the other factors affecting the diameter, as increasing the voltage or decreasing the distance leads to a decrease in the diameter of the produced nanofibres. The interaction effects between these factors were also significant. The results showed that the flow rate of the polymer solution has no significant effect on the diameter of the PAA nanofibres. In the end, the desirability function and numerical optimization were used to determine the optimum conditions for the production of nanofibres with a minimum diameter. The optimum conditions for the minimum diameter were $3 \mathrm{w} / \mathrm{v} \%$ of PAA concentration, $16 \mathrm{kV}$ of the applied voltage, $15 \mathrm{~cm}$ of the distance between nozzle and collector and $1.75 \mathrm{ml} \mathrm{h}^{-1}$ of the flow rate, and the predicted diameter for the produced nanofibres under this conditions was $262 \mathrm{~nm}$. The high agreement between the predicted diameter and the diameter of the sample produced under the optimum conditions confirms the high accuracy of the model.

\section{References}

[1] Li D and Xia Y 2004 Adv. Mater. 161151

[2] Xue J, Xie J, Liu W and Xia Y 2017 Acc. Chem. Res. 501976

[3] Hu J, Kai D, Ye H, Tian L, Ding X, Ramakrishna S et al 2017 Mater. Sci. Eng. C 701089

[4] Sharma A, Gupta A, Rath G, Goyal A, Mathur R and Dhakate S 2013 J. Mater. Chem. B 13410

[5] Hong K H 2007 Polym. Eng. Sci. 4743

[6] Chen S, Liu B, Carlson M A, Gombart A F, Reilly D A and Xie J 2017 Nanomedicine 121335

[7] Fakhrali A, Ebadi S V, Gharehaghaji A A, Latifi M and Moghassem A 2016 e-Polymers 16125

[8] Fakhrali A, Ebadi S V and Gharehaghaji A A 2014 Fibers Polym. 152535

[9] Fakhrali A, Ebadi S V, Gharehaghaji A A, Latifi M and Moghassem A 2017 Nano Hybrids and Composites 1425

[10] Heikkilä P, Taipale A, Lehtimäki M and Harlin A 2008 Polym. Eng. Sci. 481168

[11] Wang R, Liu Y, Li B, Hsiao B S and Chu B 2012 J. Membr. Sci. 392167

[12] Shokrollahi M, Morshed M, Semnani D and Rezaei B 2014 Int. J. Polym. Mater. 63161

[13] Tamura T and Kawakami H 2010 Nano Lett. 101324

[14] Wang Z-G, Wan L-S, Liu Z-M, Huang X-J and Xu Z-K 2009 J. Mol. Catal. B: Enzym. 56189

[15] Lin Q, Li Y and Yang M 2012 Sens. Actuat. B: Chem. 161967

[16] Fereydonian F, Semnani D, Morshed M, Rezaei B and Behtaj S 2015 J. Compos. Mater. 501321

[17] Huang Z-M, Zhang Y-Z, Kotaki M and Ramakrishna S 2003 Compos. Sci. Technol. 632223

[18] Patil J V, Mali S S, Kamble A S, Hong C K, Kim J H and Patil P S 2017 Appl. Surf. Sci. 423641

[19] Tan S H, Inai R, Kotaki M and Ramakrishna S 2005 Polymer 466128

[20] Ziabari M, Mottaghitalab V and Haghi A K 2010 Korean J. Chem. Eng. 27340

[21] Moroni L, Licht R, de Boer J, de Wijn J R and van Blitterswijk C A 2006 Biomaterials 274911

[22] Ding B, Yamazaki M and Shiratori S 2005 Sens. Actuat. B: Chem. 106477

[23] Kim J R, Choi S W, Jo S M, Lee W S and Kim B C 2004 Electrochim. Acta $\mathbf{5 0} 69$

[24] Asai H, Kawai T, Shimada N, Sakai T and Nakane K 2015 Sens. Actuat. B: Chem. 21476

[25] Podgórski A, Bałazy A and Gradoń L 2006 Chem. Eng. Sci. 616804

[26] Montgomery D C 2008 Design and analysis of experiments (New York: John Wiley \& Sons)

[27] Yazdanpanah M, Khanmohammadi M, Aghdam R, Shabani K and Rajabi M 2014 CCL 3175

[28] Hasanzadeh M, Hadavi Moghadam B, Moghadam Abatari M and Haghi A 2013 Bulg. Chem. Commun. 45178 
[29] Chen J-P, Ho K-H, Chiang Y-P and Wu K-W 2009 J. Membr. Sci. 3409

[30] Nasouri K, Shoushtari A M and Mojtahedi M R M 2015 Fibers Polym. 161941

[31] Naderi N, Agend F, Faridi-Majidi R, Sharifi-Sanjani N and Madani M 2008 J. Nanosci. Nanotechnol. 82509

[32] Wei L, Qiu Q, Wang R and Qin X 2018 J. Appl. Polym. Sci. 13546407

[33] Khatti T, Naderi-Manesh H and Kalantar S M 2017 Fibers Polym. 182368

[34] Amiri N, Moradi A, Tabasi S A S and Movaffagh J 2018 Mater. Res. Express. 5045404

[35] Liu A, Honma I, Ichihara M and Zhou H 2006 Nanotechnology 172845

[36] Elliott J E, Macdonald M, Nie J and Bowman C N 2004 Polymer 451503

[37] Ebadi S V, Fakhrali A, Ranaei-Siadat S O, Gharehaghaji A A, Mazinani S, Dinari M et al 2015 RSC Adv. 542572

[38] Xiao S, Shen M, Ma H, Guo R, Zhu M, Wang S et al 2010 J. Appl. Polym. Sci. 1162409
[39] Xu R, Jia M, Zhang Y and Li F 2012 Micropor. Mesopor. Mat. 149111

[40] Ebadi S V, Fakhrali A, Gharehaghaji A A, Mazinani S and Ranaei-Siadat S O 2016 Polym. Compos. 373149

[41] Jin X and Hsieh Y-L 2005 Polymer 465149

[42] Meng L, Klinkajon W, Harkin S, Supaphol P and Wnek G E 2015 Polym. Int. 6442

[43] Zhang R-Y, Zaslavski E, Vasilyev G, Boas M and Zussman E 2018 Biomacromolecules 19588

[44] McKeon-Fischer K, Flagg D and Freeman J 2011 Polymer 52 4736

[45] Sarlak N, Nejad M A F, Shakhesi S and Shabani K 2012 Chem. Eng. J. 210410

[46] Rabbi A, Nasouri K, Bahrambeygi H, Shoushtari A M and Babaei M R 2012 Fibers Polym. 131007

[47] Mohammadian M and Haghi A 2014 Bulg. Chem. Commun. 46545

[48] Meng L, Klinkajon W, K-hasuwan P R, Harkin S, Supaphol P and Wnek G E 2015 Polym. Int. 6442

[49] Lu P and Hsieh Y-L 2009 Nanotechnology 20415604 